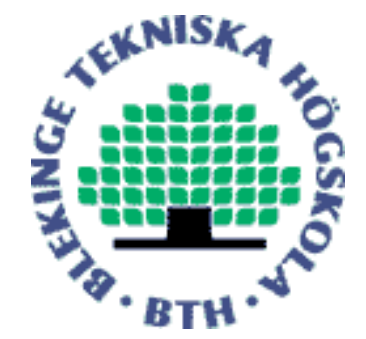

Copyright (C) 2011 IEEE.

Citation for the published paper:

Using P2P Technology to Achieve eHealth Interoperability

Yang Guo, Yan Hu, Javed Afzal, Guohua Bai

8th International Conference on Service Systems and Service Management

2011 Tian Jin, China

This material is posted here with permission of the IEEE. Such permission of the IEEE does not in any way imply IEEE endorsement of any of BTH's products or services Internal or personal use of this material is permitted. However, permission to reprint/republish this material for advertising or promotional purposes or for creating new collective works for resale or redistribution must be obtained from the IEEE by sending a blank email message to pubs-permissions@iee.org.

By choosing to view this document, you agree to all provisions of the copyright laws protecting it. 


\title{
Using P2P Technology to Achieve eHealth Interoperability
}

\author{
Yang Guo, Yan Hu, Javed Afzal, Guohua Bai \\ School of Computing \\ Blekinge Institute of Technology \\ 37179 Karlskrona, Sweden \\ xyg@bth.se, \{yahu09, jaaf07\}@student.bth.se,gba@bth.se
}

\begin{abstract}
Health is an emerging area that boosts up with advancement in Information and Communication Technology (ICT). Due to variety of eHealth solutions developed by different IT firms with no unified standards, interoperability issue has raised. In this paper, a case study in Blekinge County healthcare organizations has been conducted for understanding the contexts of eHealth interoperability issues. Then a peer-to-peer (P2P) model based on JXTA platform is implemented to solve the identified eHealth interoperability problems. According to the test result of the prototype, the suggested syntactic level interoperability among healthcare organizations has been achieved.
\end{abstract}

Keywords- eHealth; Interoperability; Peer-to-peer; JXTA

\section{INTRODUCTION}

With the rapid development in technology, world is undergoing a digital revolution in the area of Information and Communication Technologies (ICT) [1]. ICT tools are used to find, study, analyze, exchange and present information faster and more accurate. ICT has become the driving force for service organizations and citizens to access, adapt, apply and produce information interoperable [2]. One of the ICT applications in healthcare sectors is healthcare practice supported by electronic processes and communication, so called eHealth [3]. eHealth is an emerging field that covers medical informatics, public health and business that related to deliver health services and information by using ICT. eHealth makes it possible, for example, for the care providers to have fast and easy access to patient information and connect patient to care providers for home treatment, appointment and seeking of help in case emergency. It breaks the barriers among health service providers from different organizations, so they can work more closely together. ICT can also help care receivers/citizens to have better control and self-management of their own health anywhere world around. Many eHealth technologies such as Electronic Transfer of Prescription (ET), Computerized Patient Record (CPR), Electronic Health Record (EHR) and Telemedicine are widely used and they have delivered tangible benefits [4].

In Europe, efforts have been made to develop Electronic Health Record (EHR) to support the professionals to work with complex health care, and to provide accounts to simplify managing clinical work [5]. However, a major problem is interoperability among different healthcare organizations.
According to the report of US Medical Management Association and Healthcare Information and Management System Society, only $31 \%$ of doctors and $19 \%$ of hospitals are using Electronic Health Records (EHRs) because system and equipment are not interoperable [6].

In Sweden, responsibility for providing health care is decentralized to the county councils and municipal governments in some cases. In line with Swedish policy, every county council must provide residents health care at a high level, and work toward promoting good health for the entire population. Municipalities in Sweden are in charge of care for elderly people in the home or in special accommodation. Municipalities also for provide support and services for people released from hospital care as well as for school health care. In order to improve access to health care, Sweden is actively involved in cooperation across the EU. This includes collaborating on specialized care, improving patient safety and enhancing patient influence. In the other hand, the challenges of accessibility, quality, efficiency and funding are confronted in Sweden as well as other EU countries [7].

In Blekinge County Sweden, there are two main hospitals and several healthcare centers using electronic health record (EHR) in their respective systems. Hospitals use SYStem Cross while municipality healthcare centers use MAGNA CURA. These two systems are built in different technology platform. The two hospitals are interoperable with each other but they are not interoperable with municipality healthcare centers. Since these healthcare organizations are decentralized (no one is obliged to share data with others), the exchange of patient information is a problem though it is very needed from citizens (patients) perspective [8].

\section{EHEALTH INTEROPERABILITY}

\section{A. What is eHealth Interoperability?}

Healthcare interoperability is highly required among different systems in order to exchange of patient data [9]. Making systems and components interoperable will not be only a matter of speeding up information retrieval, processing and delivery among healthcare givers and hence resulting in an efficient care, it will also make the information accessible for research purpose, diagnoses, treatment and prevention of new disease [10]. 
According to Brown and Reynolds [11], interoperability on a specific task is said to exist between two applications. It means that one application can receive data from the other and perform the desired task in an appropriate and adequate manner without the need of any extra operator involvement. This definition identifies two layers of interoperability [9].

\section{1) Syntactic interoperability}

Syntactic interoperability is an application level interoperability that allowing multiple applications with different implementation languages, execution platforms and interfaces to communicate and cooperate for data exchange. Syntactic interoperability only refers to the exchange of data.

\section{2) Semantic Interoperability}

Semantic interoperability means that document is interpretable and the content is understandable by the receiver side. Semantic interoperability helps integrate data from different sources through semantic mediation. Semantic mediation is smart data discovery and integration system using knowledge based query system, which allows integrating disparate data resources.

\section{B. Challenges in eHealth Interoperability}

To achieve interoperability in eHealth area, some challenges should be faced [9].

\section{1) Interfacing}

Since interoperability among healthcare organizations is needed for exchanging information, the first problem is the interfacing problem. Interfacing is the boundary or layer at which interaction between two systems occur.

\section{2) Integration}

Combining several diverse applications into a relation for collaboration as a single entity refer to integration. This requires implementation of different standards and communication platforms.

\section{3) Accessibility}

Accessibility means that who has the right to access for patient information and at which level. There should be certain levels of accessibility like a patient can only view his record while doctor or nurse can have access to view and update his record after treatment. To cope with this challenge, a proper authentication mechanism need to be applied and certain level of accessibility should be defined.

\section{4) Security and Privacy}

Personal information should be kept private, and even may not be shared with any authority without the consensus of patient. For information security and privacy, healthcare provider should follow HIPAA (Health Insurance Portability and Accountability Act) rules, authentication procedure by allowing only authorized users also should be done.

In this paper, we focus on solving syntactic interoperability among different healthcare organizations. The semantic interoperability needs high level of standard and ontology among healthcare organizations, and will not be discussed within this paper.

\section{A CASE StUdy In BleKinge COUNTY}

To deep investigating the eHealth interoperability issues, we conducted a case study in Blekinge County, Sweden through interview.

\section{A. Purpose of interview}

The purpose of interviews is to gain knowledge about the work of existing systems and problems during communication. The main investigating topic of interview is interoperability among different healthcare centers in Blekinge County.

\section{B. Interview Planning}

For interview, we searched for contacts of the relevant personals, then sent to them brief emails about the objective of interview and requested for appointment. Three interviews were conducted based on the availability and suitability of interviewees. Two of the interviews in Ronneby from Ulf Danielsson (IT-Adminstrator, Ronneby Muncipility) and Anne Maire (Senior Nurse, Vidablick Ronneby). The 3rd interview was conducted from Jakobson (Deputy System Administrator, LANDSTINGET Karlskrona). All interviews were conducted in decent manner with full cooperation of interviewees.

\section{Interview Design}

We formulated a semi-structured interview including 30 open-ended questions in the beginning, and reduced them to 12 later on. The reason for this reduction was that some of those questions were already answered through literature or during the interviews form pervious research. Questions were designed in a way to help author for overseeing the vision of the professional about their working experience in the relevant domain. They were asked both in formal and informal mode. The whole session was mainly focused on the interoperability problem and challenges during the communication of systems.

\section{Interview Analysis}

The main objective of this interview is to study interoperability problem between SYStem Cross and MAGNA CURA, solutions to overcome this problem and discuss our proposed design. The same questions were asked to all the three interviewees in order to know different opinions about the same topic.

According to the response, health care centers in Blekinge use MAGNA CURA for healthcare management of elderly and handicapped citizen. These systems are interoperable to communicate and collaborate with each other. However, they are unable to communicate directly to SYStem Cross. There is a need for communication and collaboration between SYStem Cross and MAGNA CURA, when an elderly or handicapped patient is referred to hospitals for medical checkup or emergency. So the main purpose of communication is to exchange patient treatment summary and read some new health relevant information. 


\section{WHY PEER-TO-PEER}

\section{A. What is $P 2 P$ and JXTA?}

Peer-to-Peer is a class of applications that takes advantage of resources - storage, cycles, content, human presence available at the edges of the Internet [12]. To say it in a more clear way, P2P is a way to take advantage of previously unused resources.

According to Shirky, a problem can be solved by P2P, it must have two characters [13]:

- It treats variable connectivity and temporary network addresses as the norm.

- It gives the nodes at the edges of the network significant autonomy.

Peer-to-Peer communication can be achieved by using JXTA technology, "An open source Java based network programming and computing platform for modern distributed computing, especially for P2P networking". It designed by 'SUN Microsystems' to solve the current problems of distributed computing like interoperability, ubiquity and portability and so on. Peers in JXTA set up virtual or Ad-hoc network where each peer in the network cooperate and use resources directly behind firewall or network address translations (NATs) and even on different network [14][15]. JXTA is also a platform independent like TCP/IP and can use features of TCP/IP. JXTA does not rely on a single transport protocol as TCP/IP, but use the features provides by transport protocols. JXTA define six kinds of basic protocols such as Peer Discovery Protocol (PDP), Peer Resolver Protocol (PRP), Peer Information Protocol (PIP) and so on.

\section{B. Why P2P?}

$\mathrm{P} 2 \mathrm{P}$ has some advantages that make it a powerful tool:

Content and resources can be shared from both the center and the edge of the network. In client/server networking, content and resources are typically shared from only the center of the network.

A network of peers is easily scaled and more reliable than a single server. A single server is subject to a single point of failure or can be a bottleneck in times of high network utilization.

A network of peers can share its processor, consolidating computing resources for distributed computing tasks, rather than relying on a single computer, such as a supercomputer.

Shared resources of peer computers can be directly accessed. Rather than sharing a file stored on a central server, a peer can share the file directly from its local storage.

In this paper, we propose Atomistic P2P model for our health scenario because health is a critical issue to avoid the evolvement for central authority. Fig. 1 shows Atomistic P2P model.

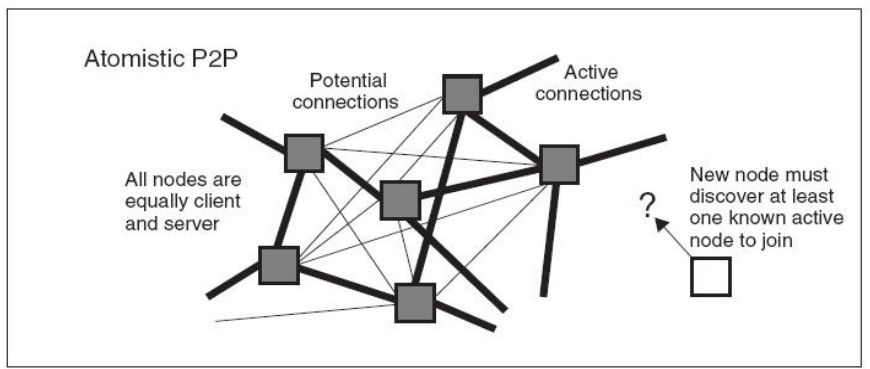

Figure 1. Automatic P2P communication model

\section{VALIDATION TO THE PROPOSED SOLUTION - PROTOTYPE DESIGN}

In our study, we design a prototype for achieving interoperability between different healthcare systems in Blekinge County. Based on the results of literature review and informal discussions, we find that JXTA may be a suitable development platform for P2P communication. JXTA is a P2Pbased collaborative approach to deal with sharing services. It is used for the different P2P systems to solve interoperability problems. JXTA is independent on operating system, network transmission technology and programming languages. It can be used in cross-platform. After the development, we test the domo with different patient data to validate our qualitative study.

\section{A. Basic framework}

In order to clarify the scope of the prototype implementation, we design a basic framework of the whole P2P workgroup communication model shown as Fig.2.

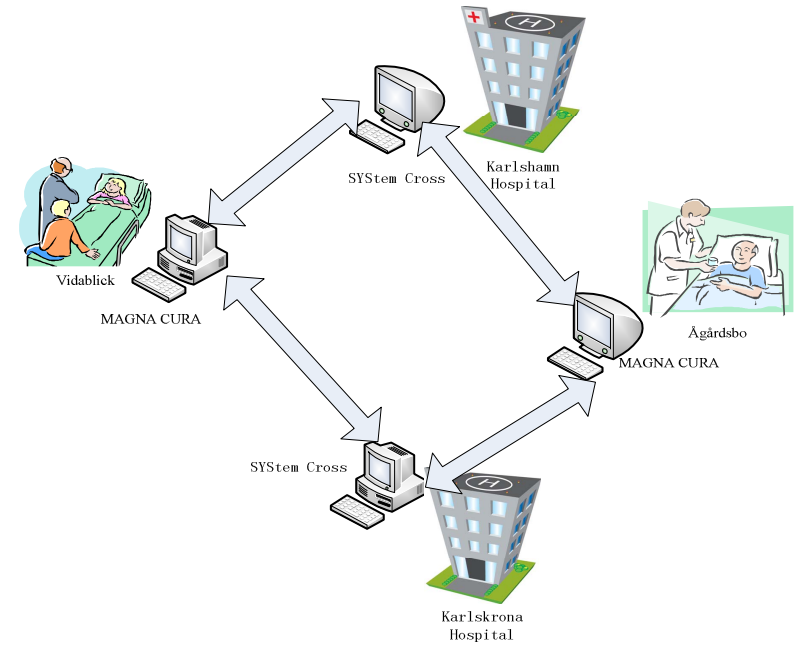

Figure 2. Basic framework of the P2P model

In this model, every healthcare center is defined as a peer. Every system is connected to the Internet. The following list the minimal basic operations.

- Share their own database to the P2P platform in every fixed time, in our case, share their database to jxta.org. 
-When one system needs to find some information of their patients, they send the request. And then if the other system has the information, they will receive the detail soon.

\section{B. Scenario for the prototype design}

For the prototype design, we assume these situations

-When the nurse at Ronneby Vidablick needs to do some medicine physical therapy, she may need some medicine information of the senior citizens from Karlskrona Hospital. So she inputs the personal number of the citizen in the third P2P communication program as a request, and then she can receive the information from the hospital's database.

- When the doctor in Karlskrona Hospital needs the physical therapy information from Ronneby Vidablick, he does the same processes to get the patient data from Vidablick's database.

Fig.3 describes the scenario of the two different systems which share the patient information.

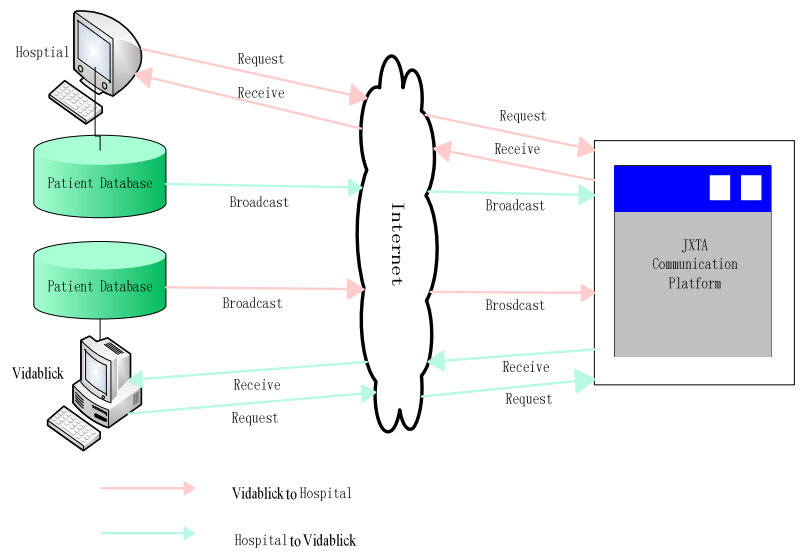

Figure 3. Share database between healthcare centers

\section{Healthcare systems simulation}

We simulated the two mentioned healthcare systems 'MAGNA CURA' and 'SYStem Cross' to validate the proposed P2P solution. The interface of 'MAGNA CURA' and 'SYStem Cross' are shown in Fig.4 and Fig.5.

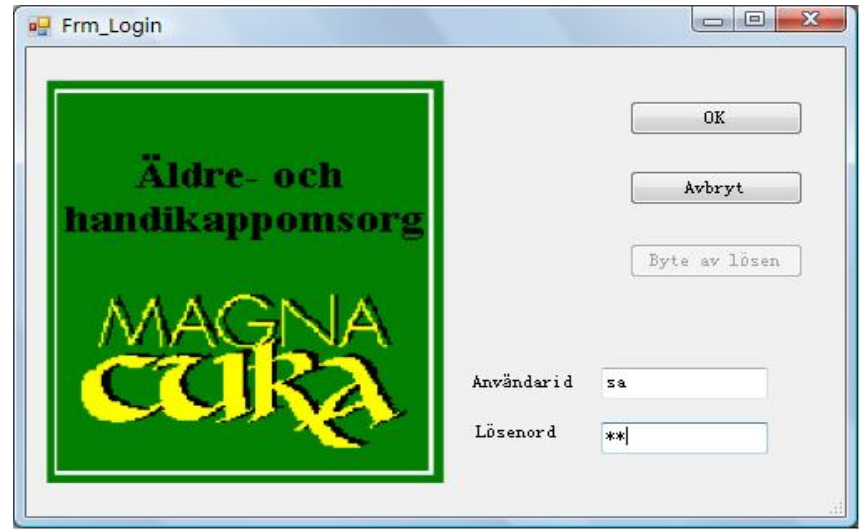

Figure 4. Healthcare system -MAGNA CURA

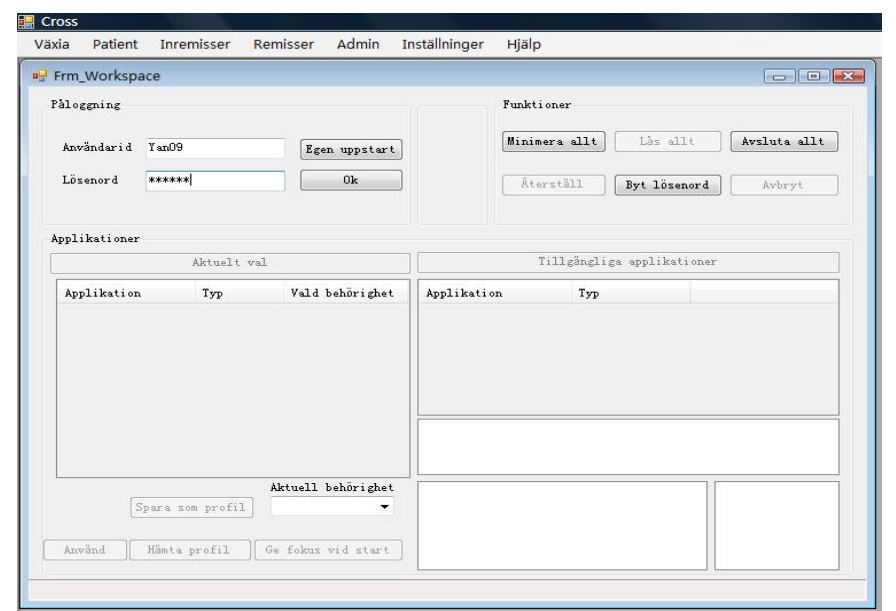

Figure 5. Healthcare system-SYStem Cross

\section{JXTA platform development}

According to "JXTA JXSE Programmers Guide 2.5" [16], we build our JATX communication platform as following steps

1. Build up JXTA data exchange architecture which is called Pipe advertisement based on XML.

2. Build JXTA group, in this case it is automatically built in Peer Group Net.

3. Set up JXTA data sending mechanism. First, we get the basic information from the database, then the Adv/ send message is created in the JXTA based data architecture. In the end, broadcast the created Adv/ send message.

4. Set up JXTA data receive mechanism. In the beginning of this step, query data is got, then query is sent and waiting for response. At last, Resolution is done if the query message is received.

\section{E. P2P communication implementation}

In the beginning of the communication, each system broadcasts their database in the third party system. In our case, all data in our peer group is sent to JXTA.org. Fig.6 shows sending data from SYStem Cross.

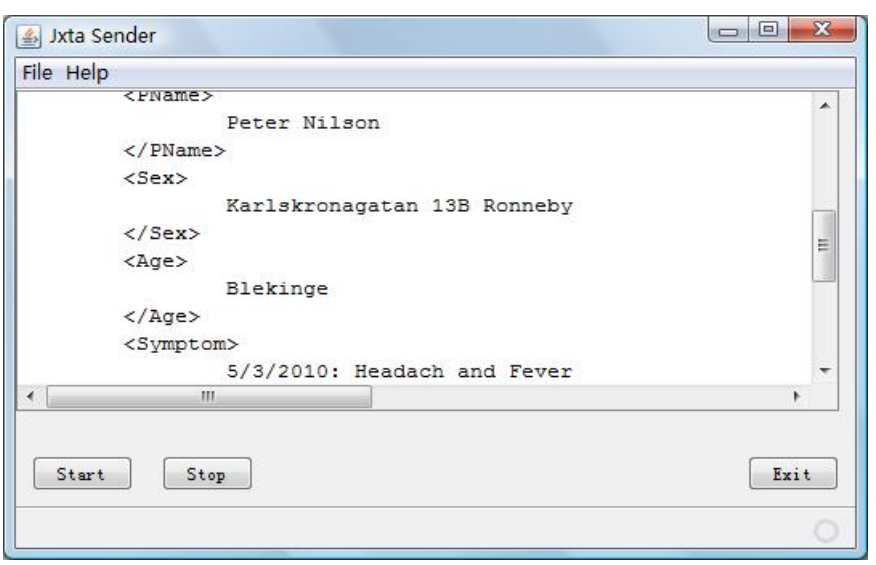

Figure 6. Sending data from SYStem Cross 
When the nurse who uses MAGNA CURA wants some medicine information from the SYStem Cross side, he/she just inputs the personal number of the elderly citizen. After some minutes, the information of that citizen is shown on the screen. As the same to the doctor of SYStem Cross, he/she inputs the personal number of the patient, the therapy records from the Vidablick is also received. Fig.7 shows receiving data in MAGNA CURA side.

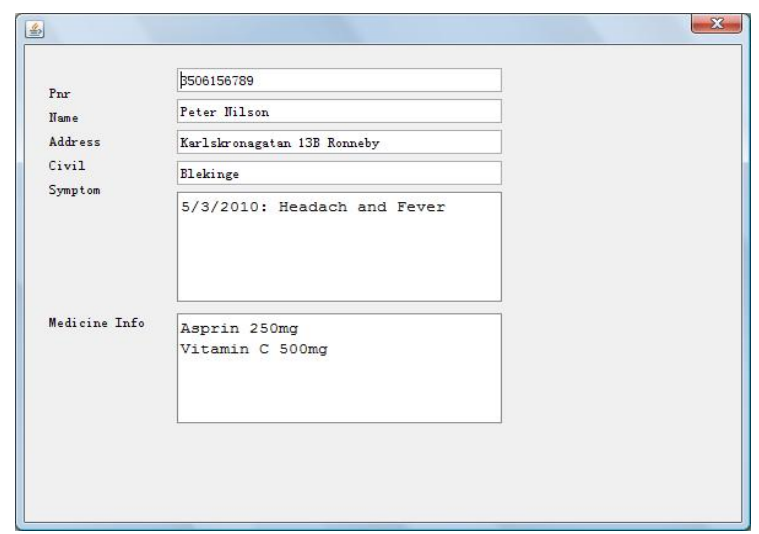

Figure 7. Receiving data in MAGNA CURA side

In the equivalent situation, if healthcare providers in SYStem Cross need to access the data stored in MAGNA CURA side, in our proposed $\mathrm{P} 2 \mathrm{P}$ model, it can be accessed easily in the same way. That's so called Peer-to-Peer.

\section{CONCLUSION AND FUTURE WORK}

The main goal of the study is to cover current interoperability gaps through proposed solution via P2P communication. We also studied many solutions that provide P2P communication but these are either proprietary or they do not provide eHelath information security. JXTA is the most important technology which is newly developed with a set of XML support and open source protocols. This study finds that it can be used to avoid these concerns. A P2P based prototype was developed with the use of JXTA technology. We tested prototype by making a group of 3 peers, and then patients' information from other peer was successfully received. In this way, we are able to achieve eHealth interoperability in Blekinge healthcare organizations at syntactic level.

However, validation of our proposed solution should be done through the real data and a survey on the targeted population for approval is needed. For the future work, we would like researchers to perform quantitative study by conducting experiments through real data in Blekinge healthcare organizations. Also, the more challenging task is semantic interoperability since eHealth is a much more complex area that needs a lot of efforts.

\section{REFERENCES}

[1] R. Mitchell F, "Information and Communication Technologies and the Global Digital Divide Technology Transfer, Development, and Least Developing Countries," Comparative Technology Transfer and Society, vol. Volume 1, no: 2, 2005, pp. pp.72-87.

[2] J. Park and S. Ram, "Information systems interoperability: What lies beneath?” ACM Trans. Inf. Syst., vol. 22, 2004, pp. 595-632.

[3] Jung M.L, Loria K. "Acceptance of Swedish e-health services". Journal of Multidisciplinary Healthcare, 2010:3, pp.55-63

[4] G. Eysenbach, "What is eHealth," Journal of Medical Internet Research, Jun. 2001.

[5] D. Sommer, eHealth for a Healthier Europe: opportunities for a better use of healthcare resources. Gartner Sverige AB, 2009.

[6] C. Bossen, "Representations at work: a national standard for electronic health records," Proceedings of the 2006 20th anniversary conference on Computer supported cooperative work, Banff, Alberta, Canada: ACM, 2006, pp. 69-78.

[7] Report, "National Strategy for eHealth • Sweden", Ministry of Health and Social Affairs, SALAR, 2006.

[8] M. Da Silveira, N. Guelfi, J. Baldacchino, P. Plumer, M. Seil, and A. Wienecke, "A SURVEY OF INTEROPERABILITY IN E-HEALTH SYSTEMS:THE EUROPEAN APPROACH”, International Conference on Health Informatics.

[9] M.E. Mansoor and R. Majeed, “Achieving Interoperability among Healthcare Organizations,” Blekinge Institute of Technology, 2010.

[10] R. Chen, "Toward Interoperability and knowledge-based Electronic Health Recording using Archetype Methodology," Linköpings universitet,, 2009.

[11] M. Eichelberg, T. Aden, J. Riesmeier, A. Dogac, and G.B. Laleci, "A survey and analysis of Electronic Healthcare Record standards," ACM Comput. Surv., vol. 37, 2005, pp. 277-315.

[12] D. S. Milojicic, V. Kalogeraki, R. Lukose, K. Nagaraja, JPruyne, B. Richard, S. Rollins, and Z. Xu, "Peer-to-Peer Computing," HP Laboratories Palo Alto HPL-2002-57 (R.1), Jul. 2003.

[13] Shirky, C. 2001. "Listening to Napster. In Oram, A., (ed) Peer-to-Peer: Harnessing the Benefits of a Disruptive Technology", O'Reilly and Associates, Inc., Sebastopol, California.

[14] “JXTA." www.jxta.dev.java.net, March 25, 2010

[15] L. Gong, "JXTA: A Network Programming Environment," IEEE Internet Computing, vol. 5, 2001, pp. 88-95.

[16] J. Group, JXTA JXSE Programmers GUide 2.5, Sun Microsystems, Inc., 2007. 